\title{
Assessing the availability of seaplane operations in the Aegean Sea
}

\begin{abstract}
The ever growing air traffic along with the need for faster passenger transportation between coastal areas, led to the quest for new air transport approaches. Seaplanes can be considered as an efficient transport solution able to connect coastal areas through seaports. To support such kind of marine operations, while comply with all safety regulations; there is a need for accurate knowledge of the local sea-state conditions and the design characteristics of the seaplanes under consideration. In this work, we have used an integrated approach to assess the suitability of the Aegean Sea for such kind of operations. The area is of great interest since it can be benefited from seaplane activities because of its complex coastline and the large number of islands. The suitability analysis has been performed with the use of seaplane design rules for three seaplane classes that differ in weight and hull length and a 10-year high resolution met-ocean dataset produced by numerical models. The analysis resulted in availability maps for the entire Aegean Sea. The latter provide information regarding the probability of occurrence of favorable weather condition for safe seaplane operations for the entire period of study and for each season separately. According to this study, coastal areas of Greece that are sheltered from the prevailing wind and wave conditions can host all three seaplane classes while as we move offshore the operability levels differentiate according to the class. Finally, these maps can be a decision tool for the design of an efficient transport network.
\end{abstract}

Volume 3 Issue 2 - 2019

\author{
C Kalogeri,' Th I Lekas, ${ }^{2}$ G Kallos' \\ 'Department of Physics, Atmospheric Modeling and Weather \\ Forecasting Group, National and Kapodistrian University of \\ Athens, Greece \\ ${ }^{2}$ Department of Aerodynamics and Flight Mechanics, Hellenic \\ Air Force Academy, Greece
}

Correspondence: Th I Lekas, Department of Aerodynamics and Flight Mechanics, Hellenic Air Force Academy, Dekelia AFB, Dekelia, Attica, Greece, Email tlekas@hotmail.com

Received: May 04, 2019 | Published: May 27, 2019

Keywords: seaplanes, safety, numerical sea-state prediction, marine operations

\section{Introduction}

Seaplanes are actually used in various missions such as firefighting or search and rescue operations. In the past, they were also used as regular passenger and light cargo carriers. However, in the post-World War II era, they had two main drawbacks compared to their land based counterparts. The first was their low aerodynamic performance, inherent to their geometry. This was the cause for the considerably lower flight speed compared to the jet airliners. The second was their low operational availability due to their inability to operate in most of the sea state conditions during water operations. These drawbacks were the main reason for their withdrawal from regular passenger carriers. Despite these downsides, such carriers are still used to connect remote areas in Canada and the U.S. This limited economic interest slowed down dramatically the development of such systems during the last fifty years and therefore most of the existing bibliography is quite old.

Seeking for new transport approaches, seaplanes are back to the forefront. To cover the needs for quick and safe connection between coastal areas and islands, seaplanes can provide a promising solution. Their safe use as passenger aircraft is strongly associated with aircraft and engine design improvements and material technology advances. If these developments are carefully applied, they can make seaplanes operationally attractive. Seaplanes can be a versatile type of transportation, covering a wide range of needs. In this context, the EUfunded Project FUSETRA (FUture SEaplane TRAffic) investigates such a possibility as one of its main objectives. ${ }^{1}$

The financial sustainability of such operations is highly related among other factors to the number of passengers that can be regularly carried and to the distance that can be covered. Towards this direction relevant studies carried out by transportation companies, which assessed different types of configurations, resulted to the fact that small seaplanes of six to seven seats are sufficient for short distances (e.g. between small islands) while the use of bigger seaplanes can be financially efficient only over longer distances, flying at altitudes higher than $6000 \mathrm{~m} .{ }^{1}$ This constraint is due to turbofans and turboprops engines efficiency that directly impacts the fuel consumption. Furthermore, flying at high altitude requires cabin pressurization, posing structural problems. Finally, special maintenance is required due to corrosion problems induced by sea salt.

Local weather conditions and sea state characteristics can seriously affect seaplane safety and/or the outcome of a mission as described, for instance, in the Flight Training Handbook. ${ }^{2}$ Safety considerations become even more stringent in passengers are considered. Therefore, the seaplane performance characteristics must comply with the constraints imposed by the local weather and sea state conditions. In particular, rough sea conditions will impose dynamic loads on the aircraft structure while additional aerodynamic issues may occur. This will eventually lead to structural failure or aerodynamic stall.

Since safety is strongly associated with the sea state and the technical characteristics of the specific aircraft, an attempt was made to identify the links between them. The main objective of this work is to develop an integrated approach for supporting seaplanes operations rather than developing a deterministic theory. In particular, this analysis aims to quantify and assess the levels of readiness for seaplane operations in the Aegean Sea. The considered approach associates the parameters describing the main characteristics of the wave field with some technical characteristics of selected seaplanes classes. This way, operational boundaries can be established. The sea-state characteristics have been provided using numerical models. The latter are useful tools for generating the necessary, high spatial 
and temporal resolution, met-ocean information for site selection and operational support. The rest of this paper is structured in four sections. In Section 2 the methodology used to estimate the operability levels for each seaplane category, over the Aegean Sea, is presented. A short description of the main wave characteristics of the Aegean Sea is given in Section 3. The main results of the analysis are discussed in Section 4 and finally a brief discussion that summarizes the main findings of this study is presented in Section 5.

\section{Methodology}

To estimate the operability levels in areas where seaplane operations are expected to take place in the near future, an availability analysis has been performed. The purpose is to establish maps of the probability occurrence of favorable operational conditions for a specific seaplane class. For this reason the crucial sea state parameters are associated with semi-empirical seaplane design rules. The wind climatology of the marine boundary layer and the associated sea state conditions are numerically described using weather and wave prediction models. Third-generation spectral wave models are widely used to describe the sea-state conditions and as next step to support marine operations. ${ }^{3}$ The modeling system used in this analysis are the wave model WAM and the atmospheric model SKIRON. ${ }^{4-8}$ WAM is a $3^{\text {rd }}$ generation phase-averaged wave model that does not provide information regarding individual waves but rather for the full directional spectra. SKIRON has been used to provide the forcing wind field to the wave model.

Both models operated in a hindcast mode for a 10-year-period (2001-2010) with $5 \times 5 \mathrm{~km}$ resolution configuration generating information of the main met-ocean parameters with a 1-hour frequency. ${ }^{9}$ This time span and spatial resolution is considered adequate to accurately describe the wave characteristics of the area. This dataset has been extensively evaluated for its accuracy, in several studies. ${ }^{9}$ For the needs of this analysis, significant wave height $(H s)$ and wave length $(\lambda)$ have been used. The estimation of the wave length has been performed as a post-process technique using the mean period $(T)$ as derived by the spectral wave model WAM based on the deep and shallow water assumptions as follows:

$$
\begin{aligned}
& \lambda=1.56 \mathrm{~T}^{2}(m) \text { deep water }\left(\frac{D}{\lambda}>\frac{1}{4}\right) \\
& \lambda=1.56 \mathrm{~T}^{2} \sqrt{\tanh \left(\frac{4 \pi^{2} D}{g T^{2}}\right)}(m) \text { shallowwater }\left(\frac{D}{\lambda}<\frac{1}{4}\right)
\end{aligned}
$$

where $\mathrm{D}$ is the sea depth.

Crucial parameters for seaplane safety during water operations are its takeoff or landing weight and the wetted length of its floats or hull (Flight Training Handbook AC 61-21A). Given the $H_{S}$ of a specific site at a specific time moment, the minimum weight that a seaplane should have in order to withstand the corresponding sea state conditions can be estimated using the equation (3) as suggested by Thurston. ${ }^{10}$

$$
W=\exp \left(\left(H_{s}+8.6424\right) / 1.25\right)
$$

where $W$ is expressed in $\mathrm{lb}$ and $\mathrm{H}_{\mathrm{S}}$ in $\mathrm{ft}$.

At the same time, the ratio of the wave length to the seaplane hull or float length should not be comprised between two and three, for structural reasons. ${ }^{11}$ It was experimentally found that in this range the vertical and angular accelerations and the structural stresses, sustained by the seaplane, attained their maximum values. Thus, according to the weight restriction and given the wave length, one can decide about the class of seaplane that can operate in the specific region under specific sea-state conditions.

The availability analysis has been performed for three seaplanes classes selected in such way to cover the entire range of the available designs. These classes are represented by three existing seaplanes that were chosen according to their size and configuration. The first category is represented by the DHC $400 \mathrm{~S}$, the second by the Bombardier CL-415 and the third by the Shimaywa HS-2. The selected aircraft are illustrated in Figure 1 and their technical characteristics are summarized in Table 1. It must be clarified that any comparison between the seaplanes that represent each class is totally out of the scope of this work.

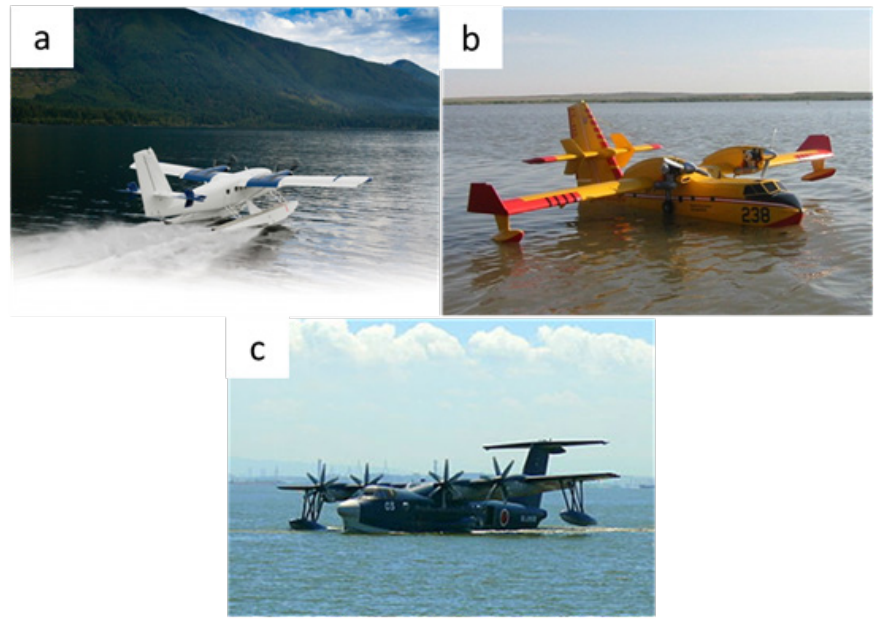

Figure I Seaplanes considered: a) DHC $400 \mathrm{~S}$, b) CL 415 and c) Shinmaywa HS-2.

Table I Seaplanes characteristics by "length" we refer to the wetted length of the floats or the hull

\begin{tabular}{llll}
\hline SEAPLANE & CONFIGURATION & LENGTH $(\mathbf{m})$ & T/OWEIGHT $(\mathbf{k g})$ \\
\hline DHC 400 S & FLOATPLANE & 9,53 & 5670 \\
BOMBARDIER CL 4I5 & FLYING BOAT & 19,82 & 17170 \\
SHIMAYWA HS-2 & FLYING BOAT & 33,15 & 43000 \\
\hline
\end{tabular}

\section{Study area-Aegean Sea}

The Aegean Sea is a semi-enclosed area with fetch regions of moderate extend located in the northern part of the Eastern
Mediterranean (Figure 2). It is characterized by extended and complex coastline, deep basins and a large number of islands. It contains large amount of bays and gulfs that can be used as seaports. Moreover, there are numerous already existing ports, harbors and marinas that can host 
seaplane operations. It is bounded to the west and north by the Greek mainland and to the east by the Asian Minor (Figure 2). The southern boundaries of the Aegean Sea are formed from west to east by the following islands: Kythira, Antikythira, Crete, Kasos, Karpathos and Rhodes. These island formations cut off the Aegean from the long period swell of the Mediterranean Sea. Cyclades complex plays the role of a boundary for the northeasterly and the southwesterly wave propagation separating the northern and the southern part of the Aegean Sea. As for the northwesterly flow the passage between Cyclades and
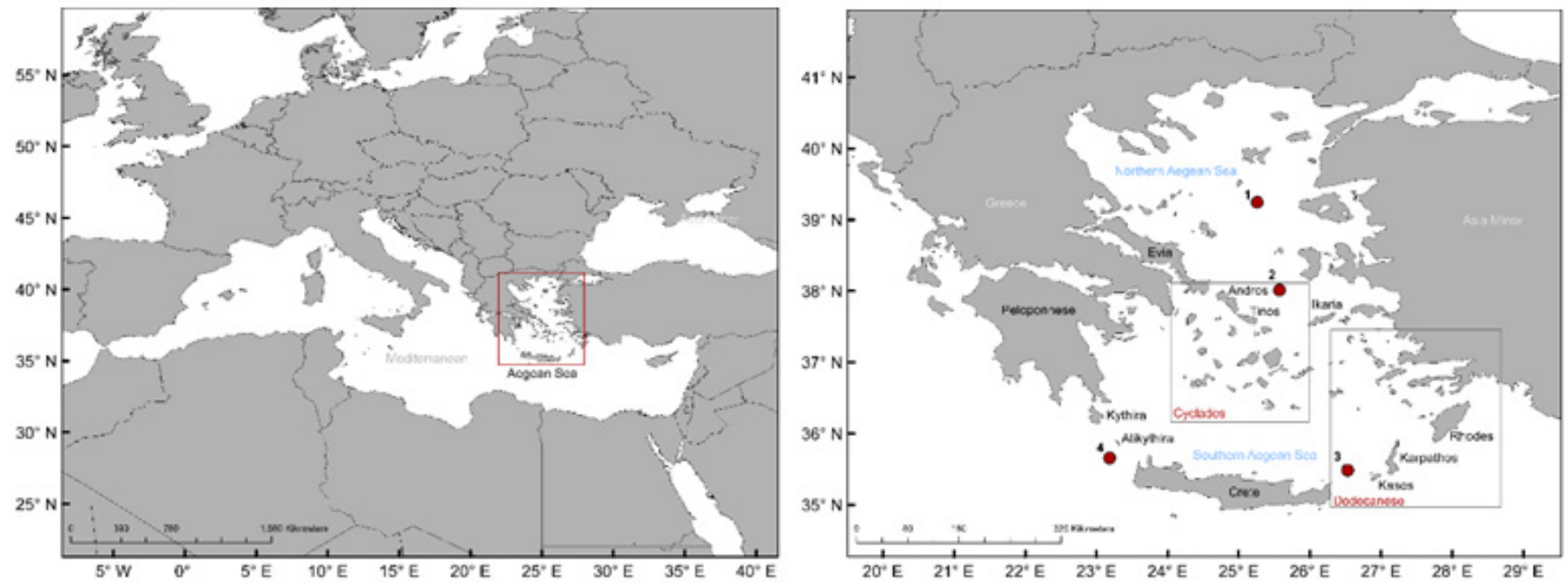

Figure $\mathbf{2}$ Area of study. The locations that have been further analyzed are represented by red dots.

During the cold period the area is influenced by low pressure systems that result to northerly and southerly waves. During the warm period the etesian winds prevail, creating mostly waves from the northern sector. ${ }^{12,13}$ Four locations of different sea-state characteristics have been selected to demonstrate the prevailing wave direction over the area. For each location the prevailing wave conditions can be retrieved by the wave rose diagrams formed using the 10 -year
Ikaria channels the flow and significant swell formations may occur. The Mediterranean swells can enter the Aegean Sea from the open passages between these islands influencing mainly the southern part of the Archipelago. The wave field can be characterized as wind driven with short swell formations due to the limited fetch of the subbasins that form the Aegean Sea. The area exhibits waves with mean significant height values less than $1.5 \mathrm{~m} .{ }^{12}$ The highest mean values are met during the winter period. hindcast data (Figure 3). In the north Aegean the prevailing wave directions are northern and north-eastern while a secondary southern is also evident. In the central Aegean the wave direction is northwestern $60 \%$ percent of the time. In the south-west Aegean the wave directions are western and north-western. Finally, in the south-east Aegean the wave directions are mostly western and the north-western and in same occasions north-eastern.

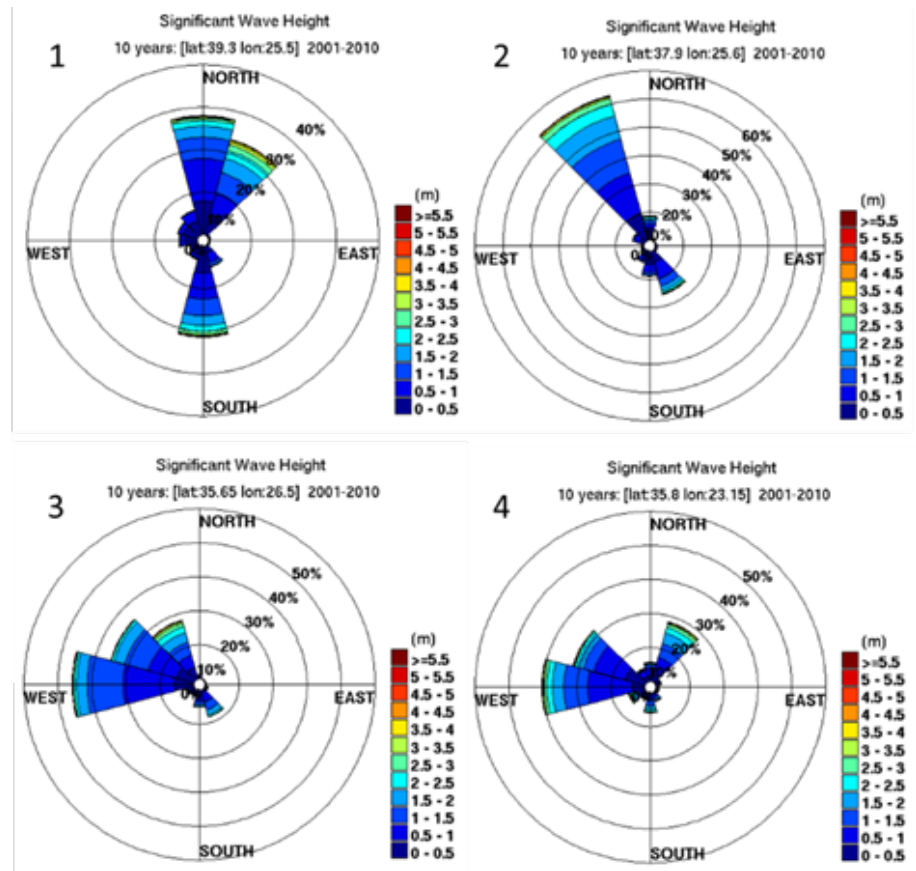

Figure 3 Directional distribution of significant wave height, for selected locations in the Aegean Sea for the period 200I-20I0. 


\section{Results}

As discussed earlier, an availability analysis for seaplane operations has been performed, for the three classes over the entire Aegean Sea. The selected aircraft to represent each class, among others characteristics, differ in weight and hull length. For each selected seaplane there is a range of favorable sea-states at which it can operate. The maps produced as a result of this analysis quantify the frequency of occurrence of such conditions for the entire area of interest. $100 \%$ operability means that the specific aircraft can operate all the time. The maps for each seaplane class for the entire 10-year period and for each season separately are presented in Figures 4-7 providing information every $5 \mathrm{~km}$.
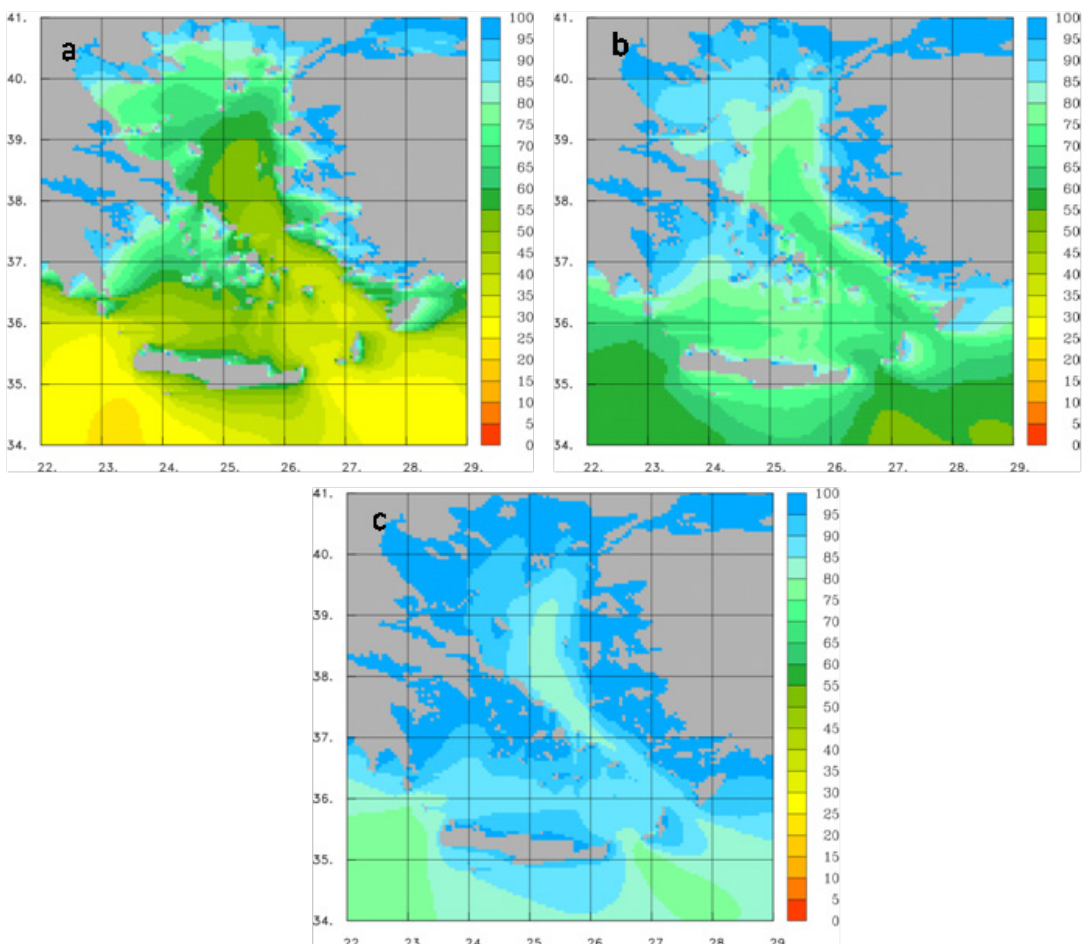

Figure 4 10-year operability levels in percentage for the considered seaplane classes represented by the following aircraft, respectively: a) DHC $400 \mathrm{~S}, \mathrm{~b}) \mathrm{CL}$ 415 and c) Shinmaywa HS-2.
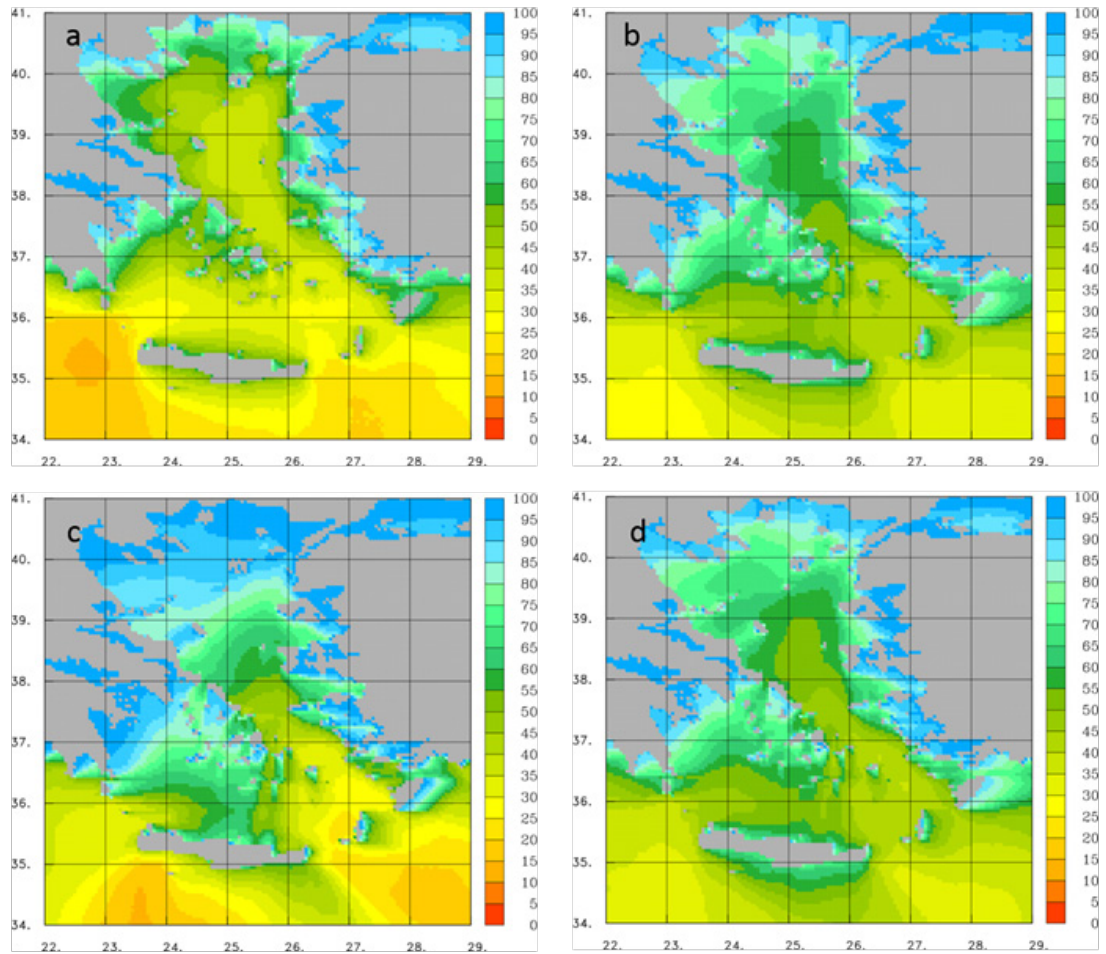

Figure 5 Seasonal operability levels in percentage for the It class represented by the DHC $400 \mathrm{~S}$ aircraft, for the 10-year period 200I-20I0: a) DecemberJanuary-February, b) March-April-May, c) June-July-August and d) September-October-November. 

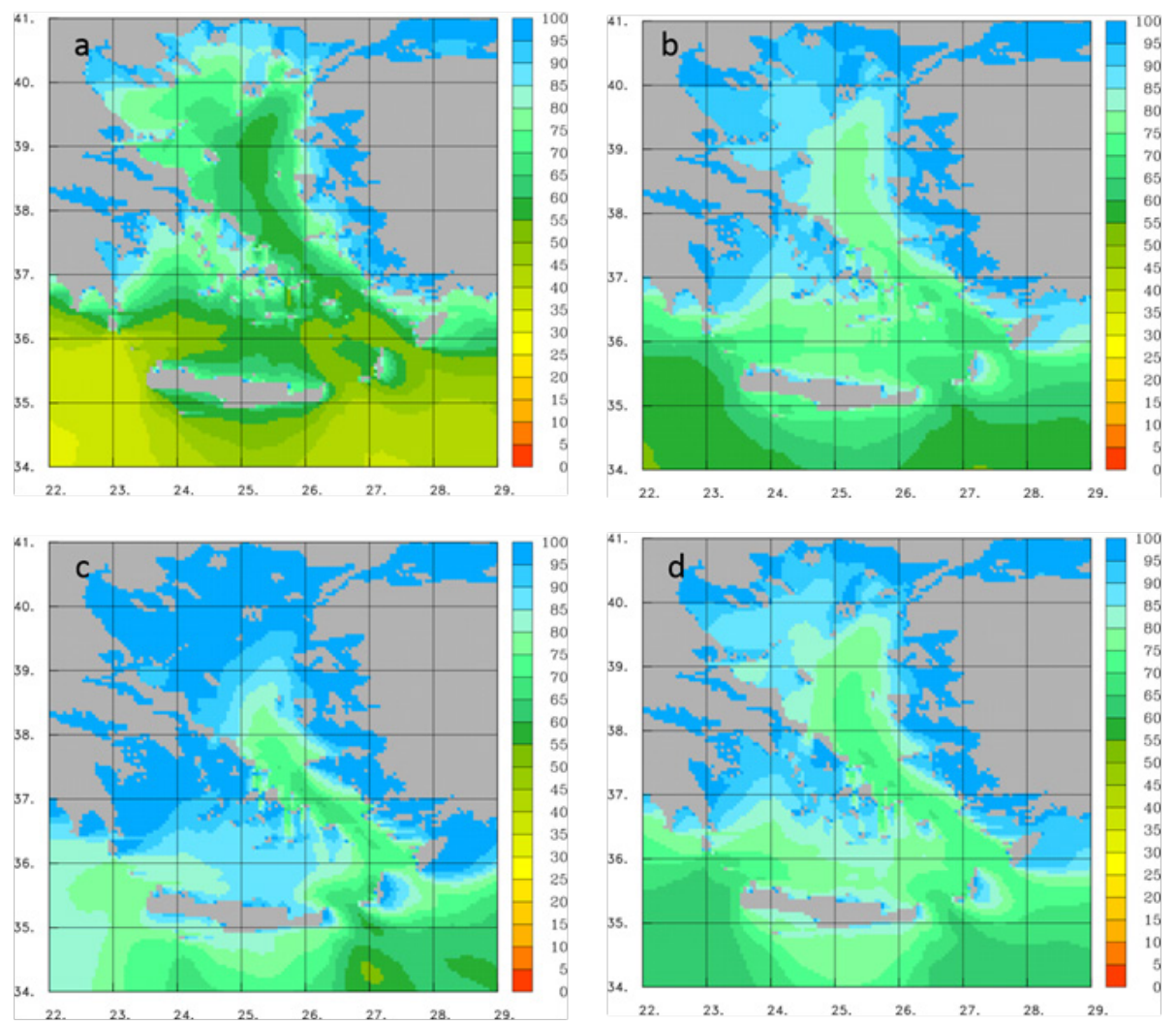

Figure 6 Seasonal operability levels in percentage for the $2^{\text {nd }}$ class represented by the CL-4I5 aircraft, for the 10-year period 200I-2010:a) December-JanuaryFebruary, b) March-April-May c) June-July-August and d) September-October-November.
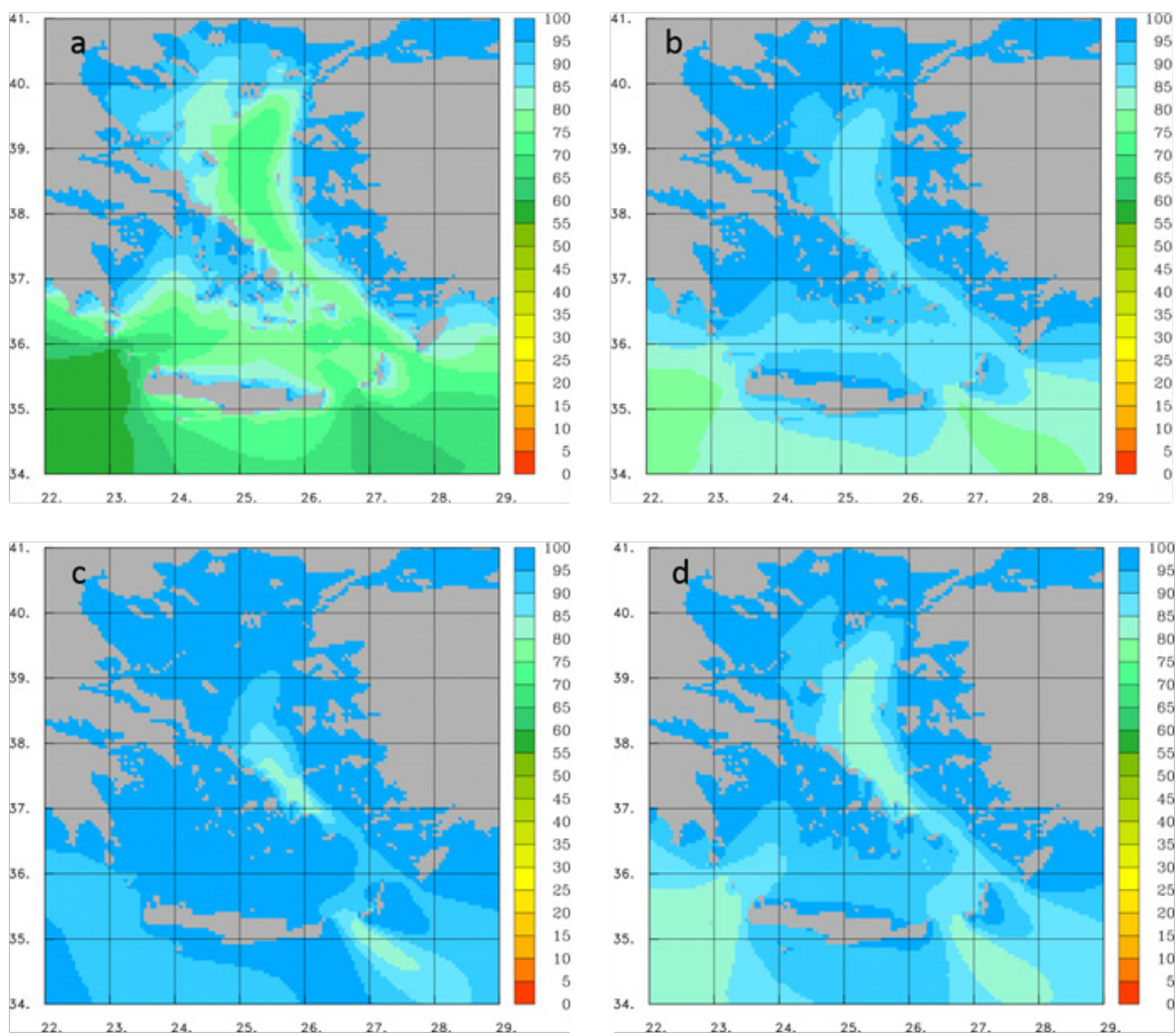

Figure 7 Seasonal operability levels in percentage for the $3^{\text {rd }}$ class represented by the Shinmaywa HS-2 aircraft, for the I0-year period 200I-20I0:a) DecemberJanuary-February, b) March-April-May c) June-July-August and d) September-October-November. 
The analysis indicates that almost all coastal areas that are sheltered from the prevailing wind and wave conditions, exhibit high operability levels for all three classes $(>90 \%)$ as illustrated in Figure 4. For the areas that are exposed to the prevailing wave systems, the percentages differentiate according to the selected seaplane class. The first class (represented by DHC $400 \mathrm{~S}$ ) exhibits the lowest values of availability, less than $80 \%$ as it can be seen in Figure 4a. The extent of the area with high operability levels ( $>90 \%)$ increases significantly as the seaplanes getting heavier and longer. Medium and heavy weight seaplanes can operate in almost all islands (Figure $4 \mathrm{~b} \&$ Figure $4 \mathrm{c}$ ). Between the two, the latter exhibits the highest operability levels over the whole domain even in windward areas.

Among the island formations, Cyclades complex has an exceptional behavior. Despite the fact, that is located in the center of the Aegean Sea, which is an area of high wind and waves, it can be considered favorable for seaplane operations (Figure 4). Even for the lighter category of sea planes (first class), there are regions that can be accessible more than $80 \%$ of the time. This is due to the fact that Evia, Andros and Tinos are physical boundaries to the prevailing N to NE wave field created on the northern Aegean fetch. For these particular islands the northern coasts are of high risk for such kind of marine operations. These areas are exposed to the dominant strong northerly wave system of the Aegean Sea revealing the lowest percentages of favorable conditions (Figures 2-4).

The least attractive areas for marine operations in the Aegean Sea are these located in the sea area between Cyclades and Dodecanese complex that extends up to the straights of Crete and Karpathos and the ones located in the straights of Crete and Peloponnese (Figure $2 \&$ Figure 4). The latter is a region exposed to eastward swells generated at a quite extended fetch starting from the Central Mediterranean while the former constitute the main channel within which the northerly winds blow over the Aegean Sea.

The smallest seaplane class (represented by DHC 400 S) despite the fact that has a rather restricted operational area, exhibits operability levels higher than $70 \%$ in most of the coastal region (Figure 4a). During the summer months when the demand for transportation is high due to the initiation of the summer vacation period, these percentages are getting higher (Figure $5 \mathrm{c}$ ).

Among the seasons, winter and summer months reveal a quite distinct behavior compared to the transient ones (Figures 5-7). The most restricted conditions are met during winter and the most favorable during summer (Figures 5a, 5c). Transient seasons on the other hand exhibit profound similarities (Figures $5 \mathrm{~b}, 5 \mathrm{~d}$ ). During summer months the northern Aegean and the eastern coasts of Peloponnese present the highest operability levels among the seasons. In contrast, the central, the southeastern and southwestern Aegean exhibit the lowest percentages, comparable to the lowest values of winter season.

In general, a rather light passenger seaplane is most suitable for short distances in the Aegean Sea (e.g. island hopping), especially during the warm period (Figures 5a, 5c). This will permit frequent low altitude flights between neighboring islands, especially small ones. Seaplanes of medium size and weight (represented by CL-415) have a broader operational area, as it is shown in Figure 4b. During the winter months (December, January, February, Figure 6a) the restriction levels are rather high, but for the rest of the year the availability is improved (Figures 6b-6d). This imposes fewer constrains in the choice of sea port locations and makes the selection of this seaplane class, for regular passenger services, much more attractive.
As it was expected, the biggest and heaviest seaplane class considered in this work (represented by Shinmaywa HS-2), can operate most of the time from almost any point of the selected geographical area, reaching levels higher than 90\% (Figure 4c). Even for this class, winter months deviate from the mean conditions (Figure 7a). During this period the central Aegean and the areas off Crete, reveal the lowest operability percentages, which are still high enough $\sim 70 \%$ (see panels of Figure 7). The high operability levels reached by the specific seaplane class, sets it to be the most appropriate for operating in the Aegean Sea. Medium and heavy weighted seaplane classes are well suited for services between long distant locations. However, these categories are represented by seaplanes that cannot provide passenger services on their current state and configuration. Under these considerations, the idea of modifying the existing (already certified) seaplane designs to passenger-carriers, could be an attractive proposal. Although, in order this to be a feasible proposal, all the financial aspects should be seriously considered.

\section{Concluding remarks}

Due to the ever growing air traffic and the environmental restrictions, new passenger air transportation solutions need to be found. One solution could be the use of seaplanes of various sizes. Like all marine operations, seaplane activities are sensitive to the sea state conditions.

In this study, an attempt was made to analyze and discuss the seastate conditions occurring in the Aegean Sea with respect to seaplane operations. The, obvious, reason is impact on safety. The Aegean area was considered because of its complex coastline that consists of a large number of island formations and many sheltered coastal areas that can be mostly benefited by such a quick and safe interconnection. To this end, an approach was used for quantifying the operability levels of specific seaplane classes, based on the crucial sea-state parameters affecting these kind of operations. The results of this analysis were used to form availability maps for the area. The availability has been established based on semi-empirical seaplane design rules and on numerical wave model outputs. They show percentages of occurrence of conditions favorable for such operations. Based on these maps, areas of high or low operability were identified for each seaplane category providing valuable information for the efficient design of a transport network in the area. The main outcomes of this study are summarized below:

a. The Aegean Sea can be considered suitable for seaplane operations due to the mild wave conditions that characterize the area most of the time.

b. The highest operability levels for all three classes can be found during the summer period where the demand is the highest for such kind of transportation because is the tourist season in Greece.

c. For the winter months large aircraft are more suitable for such kind of activities. In particular, the last and heaviest class reveals significantly high operational readiness during this period while at the same time the area that presents such favorable conditions extends significantly.

d. The most attractive areas for sea ports are these located inside bays, gulfs and sea areas sheltered from the prevailing wave field. The least attractive areas are these located between the Cyclades and Dodecanese complex and extend south-eastward 
and these located in the straights of Crete and Peloponnese. Both areas are exposed to long period swells formations that can be restrictive for such operations.

e. The two heavy classes can withstand almost all sea state conditions in the Aegean. However, their main disadvantage is that, at their current form, they cannot operate commercially.

Based on the results of this analysis and since new seaplane designs are not expected to appear in the foreseeing future, it is suggested that already existing seaplanes, especially flying boats, could be converted into passenger carriers. In any case, in order to evaluate this suggestion, all the technical and financial aspects should be examined. For this reason, a detailed technical and economic analysis is needed, focusing on three main points: The correct choice of a seaplane class for specific flight routes, the feasibility and the cost of converting existing flying boats designs into passenger carriers and finally the cost to build new sea bases of similar capacity to land airports.

\section{Acknowledgments}

None.

\section{Conflicts of interest}

Authors declare that there is no conflict of interest.

\section{References}

1. FUSETRA; 2017.

2. Flight Training Handbook (AC 61-21A), published by the US Government.
3. Niclasen BA, Simonsen K, Magnusson AK. Wave forecasts and smallvessel safety: A review of operational warning parameters. Marine Structures. 2010;23(1):1-21.

4. Janssen P. ECMWF wave modeling and satellite altimeter wave data. Elsevier Oceanography Series. 2000;63:35-56.

5. Kallos G. The Regional weather forecasting system SKIRON. In: Proceedings of the Symposium on Regional Weather Prediction on Parallel Computer Environment, Athens, Greece; 1997. 9 p.

6. Komen GJ, Cavaleri L, Donelan M. Dynamics and modelling of ocean waves. Cambridge university press; 1996.

7. Spyrou C, Mitsakou C, Kallos G, et al. An improved limited area model for describing the dust cycle in the atmosphere. J Geophys Res Atmos. 2010;115(D17):1-19.

8. WAMDI Group. The WAM model: A third generation ocean wave prediction model. J Phys Oceanogr. 1988;18:1775-1810.

9. Kalogeri C, Galanis G, Spyrou C, et al. Assessing the European offshore wind and wave energy resource for combined exploitation. Renewable Energy. 2017;101:244-264.

10. Thurston D. Design for Flying. $2^{\text {nd }}$ ed. TAB Books; 1995.

11. Magneson NJ. Site selection and preliminary planning criteria for large, high performance, water based aircraft. Thesis, Montery, California: Naval Post Graduate School; 1958.

12. Emmanouil G, Galanis G, Kalogeri C, et al. 10-year high resolution study of wind, sea waves and wave energy assessment in the Greek offshore areas. Renewable Energy. 2016;90:399-419.

13. Makris C, Galiatsatou P, Tolika K, et al. Climate change effects on the marine characteristics of the Aegean and Ionian Seas. Ocean Dynamics. 2016;66(12):1603-1635. 